\title{
How can surgical programs use peer support programs in times of crisis?
}

\author{
Leslie Scott, MD, FRCSC; Rodrick Lim, MD, FRCPC; Laura Foxcroft, MD, CCFP; P. Andrea Lum, MD, FRCPC, CCPE \\ Schulich School of Medicine and Dentistry, Western University, London, ON, Canada
}

Cite as: Scott L, Lim R, Foxcroft L, et al. How can surgical programs use peer support programs in times of crisis? Can Urol Assoc J 2021;15 (6Suppl1):36-9. http://dx.doi.org/10.5489/cuaj.7302

\section{Introduction}

COVID-19 has heightened the urgency of a long-simmering problem: physicians struggle with stress, burnout, and mental health challenges, yet they underuse support. ${ }^{1-3}$ Before COVID, reports of burnout in physicians in various specialties in Canada were highlighted in studies across multiple specialties. ${ }^{3-5}$ Similarly, studies in surgeons revealed that over $40 \%$ reported symptoms of burnout, and a survey of American surgeons alarmingly revealed one in 16 surgeons reported suicidal thoughts in the past year. ${ }^{6-10}$ A small minority sought help, citing fears of negative perception and professional repercussions. ${ }^{2,8}$ One can imagine that COVID-19 concerns of personal and family safety, vaccine access, and income instability have added significantly to the load.

\section{Important considerations for surgical specialties and burnout}

One important consideration is that surgical practice is largely procedurally based, and differs significantly from medical practice in that the therapeutic intervention is performed personally and is dependent on the skills and abilities of the surgeon. A significant stressor, specific to interventional specialties, is the catastrophic patient outcome. ${ }^{11}$ This is quite different from medical interventions, wherein a pharmaceutical agent manufactured by a third party and approved by a government agency is the actual therapeutic intervention. A surgical intervention is, in many ways, a much more personal interaction and adverse outcomes and complications are attributed on a more personal level to the surgeon, by both the patient and the surgeon.

Traditional surgical culture that has stressed individualism over collectivism and stoicism over vulnerability compounds issues around wellness in the specialty. ${ }^{11-13}$ While there has been a welcome evolution towards more collab- orative approaches to surgery, at the end of the day, there is still just one hand attached to the scalpel. Malpractice premiums are a market proxy for the burden of responsibility taken for medical care, and are consistently higher in surgical specialties. Serious adverse outcomes can result in the surgeon experiencing "second victim" syndrome. ${ }^{14,15}$ Feelings of shame, failure, self-doubt, and isolation can be overwhelming. Many surgeons do not fully recover from a serious event, and decide to modify their scope of practice or end their careers early. ${ }^{6,7}$ Surgeons surveyed after adverse events report that there was little or no support from their institution or department. ${ }^{11}$ Although the last decade has brought tangible improvements, surgical culture still stigmatizes mental health concerns, and it is still challenging to discuss adverse outcomes and medical error without blame and judgement. ${ }^{16,17}$ Given these unique considerations, one can imagine that seeking help from a non-surgical peer may pose difficulties in understanding the role that culture, guilt, and shame would play.

In addition to adverse outcomes, evolving changes in institutional structure compound stresses on surgical faculty. Ever increasing documentation demands, decreasing autonomy, and shrinking operating room $(\mathrm{OR})$ resources are significant contributors to burnout., ${ }^{9,18}$

Morbidity and mortality rounds are an important quality control and learning experience but require delicate moderation. In some centers, the process and culture of such meetings can contribute to feelings of worthlessness and humiliation. ${ }^{11,15,17}$

\section{The role of peer support programs}

Peer support programs can be a vital support and can have a positive impact on levels of hope, empowerment, and quality of life. ${ }^{19}$ They can also help create a "culture that truly values a sense of shared organizational responsibility for clinician well-being and patient safety." ${ }^{2}$

Hospital employee assistance programs (EAPs) designed for all staff do not engage physicians. Barriers include concerns about lack of confidentiality, negative impact on career, and stigma. Physicians tend to seek support from 
other physicians. ${ }^{2,20}$ As Shapiro et al conclude, "The most effective physician support system involves peers who have the unique qualification of having "been there" of having had similar experiences with stressful situations such as errors and/or litigation in the past." ${ }^{20}$ This is very important consideration in developing a support program for surgeons.

Institutional causes of burnout are much harder to address, however, peer support can alleviate some of the frustration and increase resilience. A strong and respected peer support program is not confined to one-on-one interaction but can also lobby for institutional improvements to faculty concerns.

More recently, some surgical residency programs are beginning to build a resident wellness curriculum including elements of peer support, but residents need to see it modelled and promoted by faculty. ${ }^{21-25}$ Bui et al found that the most significant factor in a review of effectiveness of resident wellness initiatives was the involvement of a faculty wellness champion. ${ }^{25}$

\section{Description of a new university-based peer support program}

The decanal office at Western University, Schulich School of Medicine \& Dentistry recognized the need to support their 2500 faculty during the COVID-19 pandemic but were challenged with short timelines, travel restrictions, and no in-person meetings due to physical distancing. Early adoption of this faculty-wide peer support program was mostly in the divisions of emergency medicine, oncology, and the department of medicine. The program engaged every clinical department to appoint interested faculty well-being leads.

Key training sessions included topics such as empathetic listening, when to seek additional support, implicit bias, professionalism, and policies and procedures around harassment and intimidation. A hands-on, small-group simulation session was developed, and anchored learnings to real-life situations based on the COVID-19 pandemic in order to best create realistic situations of anticipated conversations. A train-the-trainer model was implemented to sustain the capacity and expertise for vertical creation of local peer support within large departments. Although physicians have high degrees of communication training, key distinctions of peer support conversations were emphasized (Table 1). Professional resources of counsellors and psychologists, psychiatrists, and the provincial associations' mental health support program were identified in case people required additional support.

\begin{tabular}{ll}
\hline Table 1. Key differences/skills & required as peer supporter \\
\hline Things to do: & Things not to do: \\
\hline Empathetic listener & Compassionate colleague \\
The absence of advice and & Trying to diagnose and \\
diagnosis & advise \\
Distance from chair or reporting & Being a position of authority \\
superior & over peer \\
Confidential/privacy ensured & Lack of confidentiality/privacy \\
\hline
\end{tabular}

\section{Developing a peer support program within the surgery department}

Initially, the department had identified one peer lead with two additional peer support volunteers in a department with 108 members. The program was publicized by the department chief, and members of the department were contacted intermittently by email, offering support, contacts, and resources. The program did attempt to stimulate discussion in departmental and divisional meetings about the increased stresses for faculty during COVID-19 and the importance of addressing well-being. However, we sat back and waited...nobody called. The only uptake was by direct approach to individuals due to specific knowledge of stressful circumstances.

This point should be stressed - we do not feel that waiting for surgeons to ask for help is a successful approach. After much consideration and trial and error, a "relaunch" was then undertaken for the department of surgery, with four important points learned from our experience:

1. Validation of the program: Obtain full support and active promotion by leadership

2. Normalization of the program: In essence, an attempt to normalize it by constant exposure through better advertising and communication strategies.

3. Expansion: A plan to engage more surgeons to become peer leads and enable better peer matching by having more diversity.

4. Encourage regular "checking in" with faculty members rather than waiting for a request for support.

The department chief was asked to include the program on the agenda at each monthly executive meeting. The division chiefs were asked to nominate and endorse surgeons in their specialty to become peer leads; this validated the role and raised the profile of the program. The program was highlighted at the City Wide Surgery Meeting, with formal introduction of all the peer leads. Each subsequent City Wide Meeting will have this program on the agenda. Opportunities for academic achievement from this endeavour were stressed to surgeons, allaying the feeling that this was more time commitment without academic credit in the department. 
Peer leads chosen were NOT in positions of leadership in their divisions, removing the concern of judgement and adverse affect on career path for faculty. All were respected clinicians with relationship skills. Peer leads were chosen with diversity in mind: differing years of practice, subspecialties, gender, and race to allow surgeons to match themselves with a peer support person they feel would understand their situation.

Similar to the initiation of the above launch at the university level, surgical peer leads were offered training sessions with simulation, and instruction from psychiatry in empathetic listening. This appeared to reassure some surgical leads to join, as surgeons had privately expressed some anxiety about how they would be trained and be comfortable in the new role.

\section{How does the program currently work?}

Peer leads are encouraged to actively "reach out" to surgeons when they have direct knowledge of significant work stress; offering an opportunity to talk may bridge the difficulty surgeons have in asking for help. Referrals can also come from faculty who are concerned about an individual. Denial is a common coping mechanism, but support will still be felt from the offer and will be easier to ask for later if desired. Surgeons are free to approach any of the peer leads either from the department of surgery or from other faculty departments.

At each encounter, confidentiality is stressed. Empathetic listening and reflection are tools used to support the surgeon. The leads will be trained to look for warning signs of serious depression or suicide risk, and will have immediate access to therapeutic support available.

As mentioned above, requests for support were rare in the initial months. Physicians, in general, are reluctant to seek out help when distressed, surgeons even more so. By expanding the number and diversity of peer leads, and by increasing the validation and visibility of the program within the department, we hope the culture will evolve to allow surgeons to find it easier to seek peer support. Currently, we are up to 10 leads in our division. The initial response has been even more positive than anticipated. Likely the eager uptake for the program is stimulated by the ongoing and cumulative effects of the COVID pandemic. As we have been seeing less of each other in person in this time of enforced social isolation, the need for peer support is even more important.

We feel that with the strategy outlined above, we are much further down the road to not only acceptance and use of our surgical peer support program at this time of crisis, but that it will become a permanent and vital part of our department. We intend to collect data on attitudes to the program, usage, and benefits as we unroll it, but we feel the strategies we have learned from the development process led to a much greater chance of adoption and maintenance.

\section{Conclusions}

The COVID-19 pandemic was the crisis that catalyzed the rapid development of a peer support program designed to support a large academic faculty. The program was adapted and refocused for surgeons. Evaluation of the effectiveness of our program for the surgical faculty is ongoing. We are aiming to change culture to one of "checking in" with our peers, increasing openness and discussion to defuse stresses, decreasing feelings of isolation, and supporting recovery from critical incidents in order to begin to tackle issues of wellness and burnout in our specialty.

\section{References}

1. Lai J, Ma S, Wang Y, et al. Factors associated with mental health outcomes among healthcare workers exposed to coronavirus disease 2019. JAMA Netw Open 2020;3:e203976. https://doi.org/10.1001/jamanetworkopen.2020.3976

2. Hu YY, Fix ML, Hevelone ND, et al. Physicians' needs in coping with emotional stressors: The case for peer support. Arch Surg 2012;147:212-7. https://doi.org/10.1001/archsurg.2011.312

3. Wallace J, Lemaire J, Ghali W. Physician wellness: A missing quality indicator. Lancet 2009;374:171421. https://doi.org/10.1016/S0140-6736(09)61424-0

4. Canadian Medical Association. CMA National Physician Health Survey: A national snapshot October 2018. Available at: https://www.cma.ca/sites/default/files/2018-11/nph-survey-e.pdf. Accessed Aug. 3, 2020.

5. Lim R, Aarsen KV, Gray S, et al. Emergency medicine physician burnout and wellness in Canada before COVID19: A national survey. CJEM 2020;22:603-7. https://doi.org/10.1017/cem.2020.431

6. Shanafelt T, Sloan J, Satele D, et al. Why do surgeons consider leaving practice? J Am Coll Surg 2011;212:421-2. https://doi.org/10.1016/i.jamcollsurg.2010.11.006

7. Shanafelt TD, Balch CM, Bechamps $G J$, et al. Burnout and career satisfaction among American surgeons. Ann Surg 2009;250:463-71. https://doi.org/10.1097/SLA.0b013e318lac4dfd

8. Shanafelt TD, Balch CM, Dyrbye L, et al. Special report: Suicidal ideation among American surgeons. Arch Surg 2011;146:54-62. https://doi.org/10.1001/archsurg.2010.292

9. Campbell DA, Sonnad SS, Eckhauser FE, et al. Burnout among American surgeons. Surgery 2001;130:696-705. https://doi.org/10.1067/msy.2001.116676

10. Dimou FM, Eckelbarger D, Riall TS. Surgeon burnout: A systematic review. J Am Coll Surg 2016;222:1230-9. hitps://doi.org/10.1016/i.jamcollsurg.2016.03.022

11. Srinivasa S, Gurney J, Koea J. Potential consequences of patient complications for surgeon well-being: A systematic review. JAMA Surg 2019;154:451-7. https://doi.org/10.1001/jamasurg.2018.5640

12. Balch $C M$, Freischlag JA, Shanafelt TD. Stress and burnout among surgeons: Understanding and managing the syndrome and avoiding the adverse consequences. Arch Surg 2009;144:371-6. https://doi.org/10.1001/archsurg.2008.575

13. Luu S, Patel P, St-Martin L, et al. Waking up the next morning: Surgeons' emotional reactions to adverse events. Med Educ 2012;46:1179-88. https://doi.org/10.1111/medu.12058

14. Wu AW. Medical error: The second victim. The doctor who makes the mistake needs help too. BMJ (Clinical research ed) 2000;320:726-7. https://doi.org/10.1136/bmi.320.7237.726

15. Marmon LM, Heiss K. Improving surgeon wellness: The second victim syndrome and quality of care. Semin Pediatr Surg 2015;24:315-8. hitps://doi.org/10.1053/i.sempedsurg.2015.08.011

16. Schwappach DLB, Koeck CM. What makes an error unacceptable? A factorial survey on the disclosure of medical errors. Int J Qual Health Care 2004;16:317-26. https://doi.org/10.1093/intqhc/mzh058

17. Orlander ID, Barber TW, Fincke BG. The morbidity and mortality conference: The delicate nature of learning from error. Acad Med 2002;77:1001-6. https://doi.org/10.1097/00001888-20021000000011 
18. Ravenhearst D. A surgeon's account of physician burnout and depression. The Varsity, August 11, 2019.

19. Bellamy C, Schmutte T, Davidson L. An update on the growing evidence base for peer support. Ment Health and Soc Ind 2017;21:161-7. https://doi.org/10.1108/MHSI-03-2017-0014

20. Shapiro J, Galowitz P. Peer support for clinicians: A programmatic approach. Acad Med 2016;91:12004. https://doi.org/10.1097/ACM.0000000000001297

21. Williams-Karnesky RL, Greenbaum A, Paul JS. Surgery resident wellness programs: The current state of the field and recommendations for creation and implementation. Adv Surg 2020;54:149-71. https://doi.org/10.1016/i.yasu.2020.05.005

22. Wolfe $S Q$, West JL, Hunt MA, et al. A comparison of the existing wellness programs in neurosurgery and institution champion's perspectives. Neurosurgery 2019;84:1149-55. https://doi.org/10.1093/ neuros/nyy418

23. Kemp MT, Rivard SJ, Anderson S, et al. Trainee wellness and safety in the context of COVID-19: The experience of one institution. Acad Med 2020. Epub ahead of print. https://doi.org/10.1097/ ACM. 0000000000003853

24. Abrams MP. Improving resident well-being and burnout: The role of peer support. I Grad Med Educ 2017;9:264. https://doi.org/10.4300/JGME-D-16-00805.1

25. Bui AH, Ripp JA, Oh KY, et al. The impact of program-driven wellness initiatives on burnout and depression among surgical trainees. Am J Surg 2020;219:316-21. https://doi.org/10.1016/i. amisurg.2019.10.027

\section{Orphan references}

1. Squiers JJ, Lobdell KW, Fann JI, et al. Physician burnout: Are we treating the symptoms instead of the disease? Ann Thorac Surg 2017;104:1117-22. https://doi.org/10.1016/i.athoracsur.2017.08.009

2. Rothenberger DA. Physician burnout and well-being: A systematic review and framework for action. Dis Colon Rectum 2017;60:567-76. https://doi.org/10.1097/DCR.0000000000000844

Correspondence: Dr. Leslie Scott, Schulich School of Medicine and Dentistry, Western University, London, 0N, Canada; Leslie.Scott@hsc.on.ca 